\title{
Thermal instability of electrolytic capacitor bank used for gas puff valve
}

\author{
P. M. Bellan a) \\ Thomas J. Watson Sr. Laboratories of Applied Physics, California Institute of Technology, \\ Pasadena, California 91125
}

(Received 12 March 2002; accepted for publication 15 April 2002)

\begin{abstract}
It is shown that self-heating of electrolytic capacitors causes the output current of a capacitor bank to increase with successive shots even though the charge voltage is held constant. Self heating of only $10{ }^{\circ} \mathrm{C}$ can cause a near tripling in the gas output of the gas puffing valves commonly used in spheromak research. By using metallized polypropylene film capacitors instead of electrolytic capacitors the reproducibility is substantially improved (the shot-to-shot variation in gas output is reduced to be $<0.5 \%$ ). (C) 2002 American Institute of Physics. [DOI: 10.1063/1.1488677]
\end{abstract}

\section{INTRODUCTION}

Pulsed plasma experiments do not have a good reputation for reproducibility. There is a lexicon of "good shots" versus "bad shots" and it is typically assumed that the large shot-to-shot variation is due to intrinsic randomness in the plasma. However, it is possible that in some situations, poor reproducibility is not the fault of the plasma but rather of the system producing the plasma. This article identifies electrolytic capacitors as a component that can lead to a great deal of irreproducibility. This identification was done in the context of fast gas puff valves, but the results should also be relevant to other pulsed systems powered by electrolytic capacitor banks. The problem discussed here is probably more serious for gas puff valves than for other systems because gas valve output is a very sensitive function of the current used to pulse the valve and because plasma behavior can be a very sensitive function of gas valve output.

Gas puff valves have been used on many spheromak and spheromak-related experiments. ${ }^{1-9}$ These valves provide a brief pulse of high pressure gas localized between electrodes bounding the plasma formation region. The distance between the electrodes is called $d$ and the required voltage for the neutral gas of pressure $P$ to break down and form plasma is a function of $P d$. Curves of breakdown voltage versus $P d$ are called Paschen curves and have a minimum at a $P d$ which depends on the gas species. ${ }^{10}$ The required voltage for breakdown rapidly increases and then becomes infinite if $P d$ is moved to the left of the Paschen minimum, but the required voltage for breakdown increases gradually if $P d$ is moved to the right of the Paschen minimum. Hydrogen is the gas of most interest for spheromaks; for hydrogen the Paschen minimum occurs at $P d \simeq 2 \mathrm{mT} \mathrm{cm}$ and breakdown becomes impossible for $P d<0.8 \mathrm{mT} \mathrm{cm}$. At the Paschen minimum for hydrogen, only $300 \mathrm{~V}$ are required for breakdown. Breakdown is thus an extremely sensitive function of gas pressure if $P d$ is near the Paschen minimum or if the applied voltage is only slightly higher than the voltage required for breakdown at a given $P d$. Once the gas has broken down

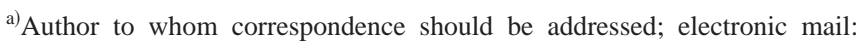
pbellan@its.caltech.edu
}

and formed plasma, MHD forces accelerate the plasma out of the formation region to fill the available volume. Gas puff valves allow the volume-averaged plasma density to be much lower than the density required for breakdown. Having a much lower average density than the density required for breakdown allows creation of hot, fully ionized plasmas, a necessity for fusion applications.

A typical fast gas puff valve uses a pulsed coil to diamagnetically repel a conducting diaphragm which functions as the valve seal. ${ }^{11-22}$ Closing of the valve is achieved by springs, inlet gas pressure, or mechanical deformation of the diaphragm. In some cases a plenum is used to meter a precise quantity of gas.

Opening times for these valves are 25-100 $\mu$ s and gas throughputs are of the order of $1 \mathrm{~cm}^{3}$ at pressures of one to a few atmospheres. Since the gas flow is supersonic, these valves are also known as pulsed supersonic valves. The shock waves produced by the supersonic flow have been used to entrain fine particles. ${ }^{23}$

Reproducibility of the gas puff can affect the reproducibility of spheromak performance. For example, shot-to-shot variation in the amount of gas injected by the gas valve could cause shot-to-shot variation of the plasma density and, in circumstances where the applied voltage is only marginally higher than the Paschen curve requirements, could also cause a substantial temporal jitter of the breakdown time. Considerable attention is therefore given to having precise mechanical construction and a precise electrical pulse. Despite this care in construction and operation, it has been previously noted that these valves can exhibit a substantial upward drift in gas throughput during the course of a day. ${ }^{24,25}$ This drift was conjectured to be due to either valve heating or slight variations in room temperature.

The purpose of this article is to report the results of an investigation into the cause of this undesirable variation in valve throughput (valve transient response will be discussed elsewhere). These results show that the variation in throughput is indeed of thermal origin, but is not due to room temperature variations or valve heating. Valves of the design sketched in Fig. 1 were used. These valves were constructed with good mechanical tolerances and were energized by a capacitor bank which was charged by a commercial capacitor 


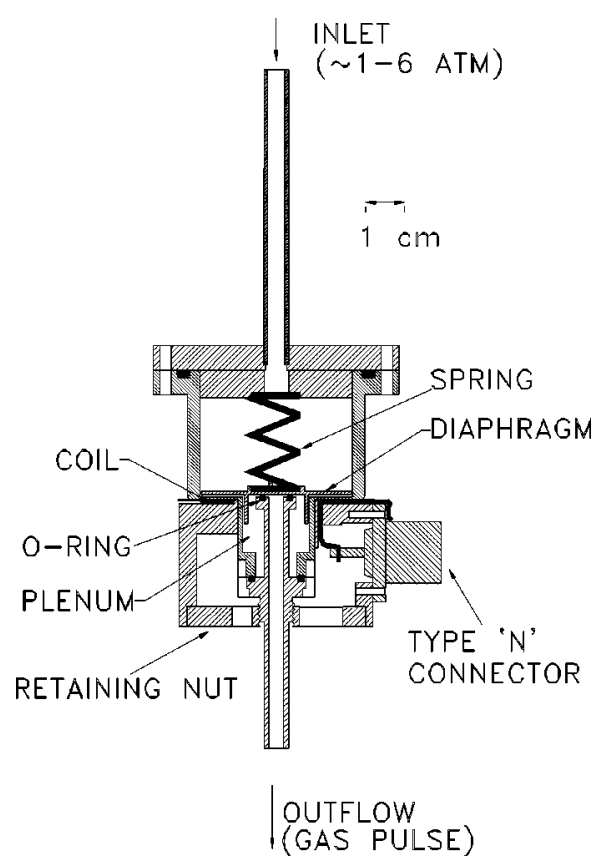

FIG. 1. Fast gas valve: Pulsed current from capacitor bank energizes coil. Magnetic pressure from current in coil diamagnetically repels diaphragm and allows gas to pass from plenum into outflow tube. Spring seats diaphragm and retaining nut presses coil against valve body.

charging supply having $0.02 \%$ voltage regulation (Ultravolt Corp. Model 1C-P24). Despite this excellent voltage regulation, careful measurements of the pressure pulse provided by the gas valve showed that the magnitude of the pressure pulse crept upwards by $\sim 0.8 \%$ per shot, i.e., increased approximately $50 \%$ over a series of 50 successive shots. The capacitor bank voltage and the inlet gas pressure were identical for these 50 shots and the interval between shots was $1 \mathrm{~min}$.

\section{IDENTIFICATION OF CAUSE OF IRREPRODUCIBILITY}

The first possibility considered was to see whether the upwards drift in valve throughput was due to a thermal problem in the gas valve itself. This seemed unlikely because heating of electromechanical components was expected to reduce rather than increase gas throughput (e.g., a more resistive valve coil would provide less current for repelling the diaphragm or a hot diaphragm would be less diamagnetic). Thermocouple measurements of the temperatures of the diaphragm, the gas inside the valve, and the valve body all showed that the temperature change for these components was less than $0.5^{\circ} \mathrm{C}$ over a large number of shots. This slight variation in temperature seemed insufficient to explain the $50 \%$ increase in the gas output over 50 shots. Another possibility was that the valve o-ring developed a compression set after many shots; this possibility was eliminated by showing that there was no difference when a low compression set Dupont Kalrez o-ring was used instead of the conventional viton o-ring.

Attention then turned to the capacitor bank and it was discovered that the problem was caused by the capacitors. The capacitor bank consisted of eighteen generic photoflash

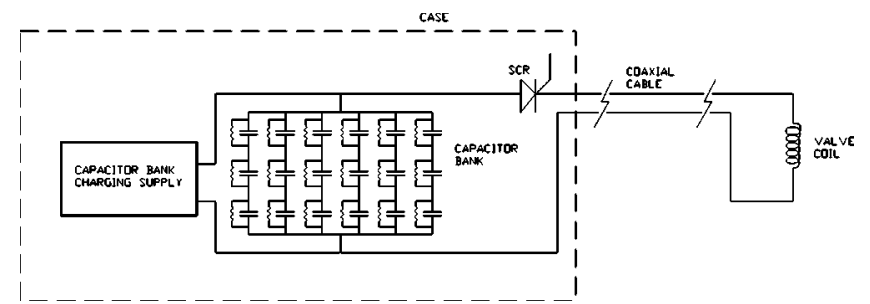

FIG. 2. Circuit of electrolytic capacitor bank; all components in the same case.

type electrolytic capacitors connected in the series-parallel configuration shown in Fig. 2. Each capacitor was rated at $200 \mu \mathrm{F}$ and $360 \mathrm{~V}$; the three-in-series arrangement was to achieve a $1 \mathrm{kV}$ rating for the capacitor bank. Resistors of 350 $\mathrm{k} \Omega$ were connected across each capacitor to ensure equal division of voltage across the capacitors. The resistors also functioned as bleeder resistors to discharge the capacitors in a few seconds when the power supply was turned off. The capacitors were mechanically mounted on an acrylic base using insulating cement (General Electric RTV) so as to have good high voltage insulation. For operator safety and convenience, the capacitors and charging supply were mounted inside a small aluminum case (shown as a dashed line in Fig. 2) which had various connectors, switches, and a digital panel meter. The total bank capacitance was $400 \mu \mathrm{F}$ and the energy stored at a nominal charging voltage of $400 \mathrm{~V}$ was $32 \mathrm{~J}$.

The mundane construction details of this modest capacitor bank are provided here because they resulted in an important and unexpected consequence. The mounting arrangements of the capacitors meant that they were not only well insulated electrically, but also were well insulated thermally. The bleeder resistors together provided about $900 \mathrm{~mW}$ of heat input at the nominal $400 \mathrm{~V}$ charging voltage and the capacitor charging power supply also provided heat input to the case interior. Electrolytic capacitors are quite lossy and so, when discharged, a significant fraction of the stored energy is dissipated internally rather than in the load. This internal dissipation is conventionally represented by an equivalent series resistance (ESR) and causes heating of the capacitor. Because the capacitors were well insulated thermally from their surroundings, they can develop an appreciable temperature rise even for a rather small energy input.

Initial proof that the electrolytic capacitor bank was the cause of the problem was obtained by firing the capacitor bank into a $60 \mathrm{~m} \Omega$ dummy load resistor which simulated the load presented by the valve. Precision measurements of the voltage across this dummy load showed that the peak current from the capacitor bank consistently increased by about $0.14 \%$ per shot (specifically, $2.5 \mathrm{~A}$ increase out of $1750 \mathrm{~A}$ total). Because any increase in current could not be the result of heating the dummy load, it was realized that the increase must be due to some other cause.

It was conjectured that this tiny, but consistent shot-toshot increase in peak current could be due to heating of the capacitors and so the capacitor temperature rise was monitored using a thermocouple (Omega Engineering SA1-J) adhesively mounted to the side of one of the electrolytic ca- 
pacitors. In standby conditions (i.e., no pulsing, but bank charged to $400 \mathrm{~V}$ ), it was found that the capacitor temperature would increase about $5{ }^{\circ} \mathrm{C}$ above room temperature in 1 h. On the other hand, if the bank was charged to $400 \mathrm{~V}$ and pulsed once per minute, the capacitor temperature increased by about $10^{\circ} \mathrm{C}$ above room temperature over 50 shots. A pulse rate of once per minute corresponds to $520 \mathrm{~mW}$ average power from the capacitor bank.

These powers and temperature rises are so modest that they normally would not merit attention or cause concern. However, even though modest, the capacitor temperature rise turns out to be critical for electrolytic capacitors. Since the temperature measurement is on the outside of the capacitor case, the interior temperature of the capacitor would be significantly higher than the measured temperature and so the measured temperature rise is a conservative estimate of what is happening inside the capacitor. For pulse (i.e., photoflash) electrolytic capacitors the ESR turns out to be a strongly decreasing function of temperature. Because the gas valve coil purposely has very low resistance in order to maximize current, most of the circuit resistance consists of the capacitor ESR. The capacitor bank is effectively discharging into a near short-circuit load and so the peak current of the discharge circuit is determined mainly by the capacitor bank ESR. Furthermore, the gas valve itself depends sensitively on coil current $I$ because the diaphragm displacement is proportional to the magnetic pressure which scales as $I^{2}$. It is also expected that gas flow through an opening increases nonlinearly with the size of the opening because the flow is turbulent at small openings but laminar at larger openings. Scans of gas throughput as a function of valve current showed that the throughput would approximately double with a $10 \%-20 \%$ increase in coil current.

\section{SYSTEMATIC MEASUREMENTS OF THERMAL CREEP}

Because it was too tedious to manually follow these temperature rises of $\sim 0.1{ }^{\circ} \mathrm{C}$ per shot, an automated CAMACbased system was assembled which pulsed the system in a controlled sequence over a long time. Typically, the electrolytic capacitor bank was charged to $400 \mathrm{~V}$ for each shot with a $1 \mathrm{~min}$ interval between shots. Fifty shots would be fired and then there would be a waiting time of $4 \mathrm{~h}$ so that the capacitors would cool. This sequence was repeated four times. This protocol was chosen to simulate typical laboratory routine (e.g., operating for a few hours in the morning, going to lunch, operating for a few hours in the afternoon, turning off the equipment overnight). In some 200 shot runs the capacitors were left fully charged during the $4 \mathrm{~h}$ wait interval and in some runs the capacitors were not charged during the $4 \mathrm{~h}$ wait interval (this choice was software selectable).

Helium gas was used so that the system could be safely left unattended overnight (normally hydrogen gas is used for spheromaks). The gas valve output went to a 30 liter vacuum chamber and the pressure rise in the chamber was determined using an MKS Instruments Baratron capacitance manometer (Model 622A02 TAE) which measured the chamber

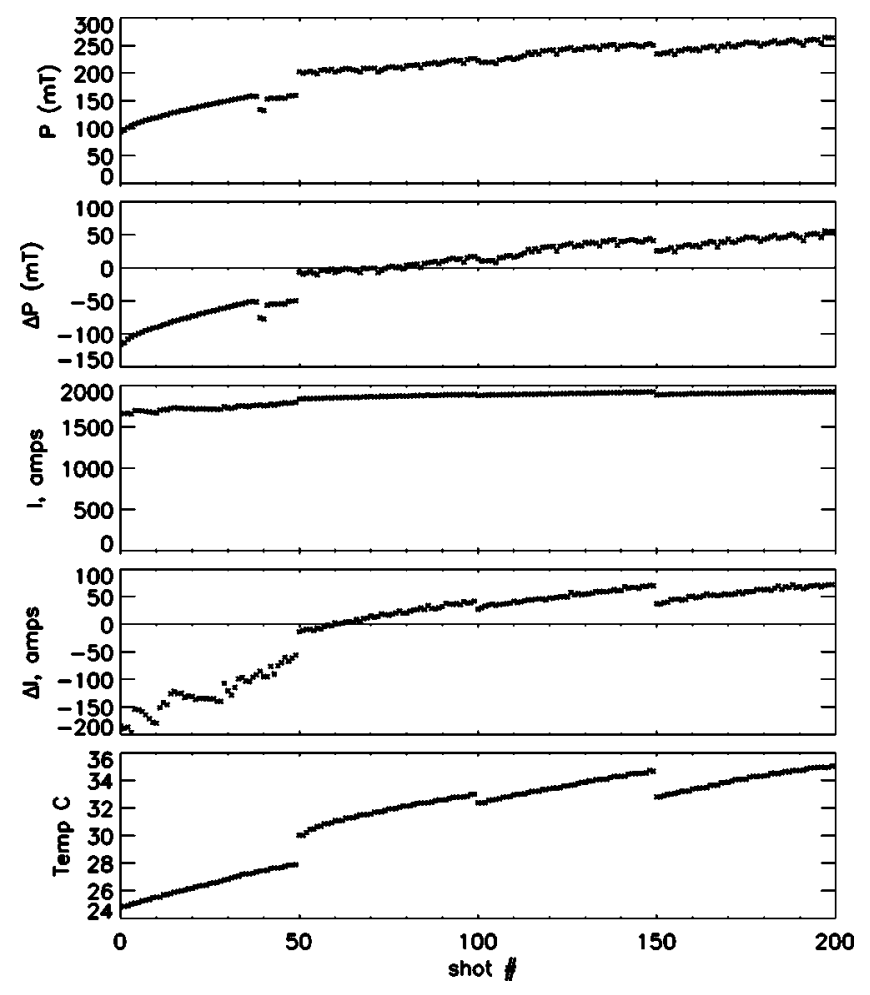

FIG. 3. Electrolytic capacitor in the same case as charging supply, meter and SCR: 200 shot sequence consisting of four sets of fifty shots with a $4 \mathrm{~h}$ wait between sets (capacitors charged during this wait). There is a $1 \mathrm{~min}$ interval between successive shots in each set. Capacitors are charged to 400 V. From top to bottom: vacuum tank pressure rise $P$ associated with a shot, $\Delta P$ the deviation in pressure rise from the mean pressure rise of the 200 shots, peak current $I$ from capacitor bank, $\Delta I$ the deviation in peak current from the mean peak current of the 200 shots, temperature measured by a thermocouple attached to side of a capacitor.

pressure immediately before a shot and then $300 \mathrm{~ms}$ after a shot. The output current of the capacitor bank was measured by a Rogowski coil. The Rogowski coil voltage was recorded using a Transiac 2008 transient digitizer running at 10 megasamples per second and this signal was then integrated numerically. The capacitor temperature obtained from the thermocouple was recorded after each shot using a Standard Engineering AD-112 12 bit CAMAC module.

Figure 3 shows the results of a typical sequence using these electrolytic capacitors. During the first 50 shots $(1 \mathrm{~min}$ interval between shots) the temperature, peak current, and chamber pressure rise all increase with successive shots. There is a $4 \mathrm{~h}$ wait between the 50th and the 51 st shot so that the capacitor temperature decreases, but remains higher than the cold start temperature because of the heat input from the bleeder resistors and the standby dissipation of the charging supply (the capacitors are held at $400 \mathrm{~V}$ during the $4 \mathrm{~h}$ standby periods for this particular 200 shot sequence). The temperature then increases again on the 51st to the 100th shot as does the current and chamber pressure rise. There is another $4 \mathrm{~h}$ wait after the 100th shot, and the system cools down again. This pattern repeats itself. These results show that when the electrolytic capacitors heat up by just $10^{\circ} \mathrm{C}$, their ESR decreases by an amount such that the capacitor bank current increased by about $16 \%$ and the gas output of the valve increases by about $250 \%$ ! 


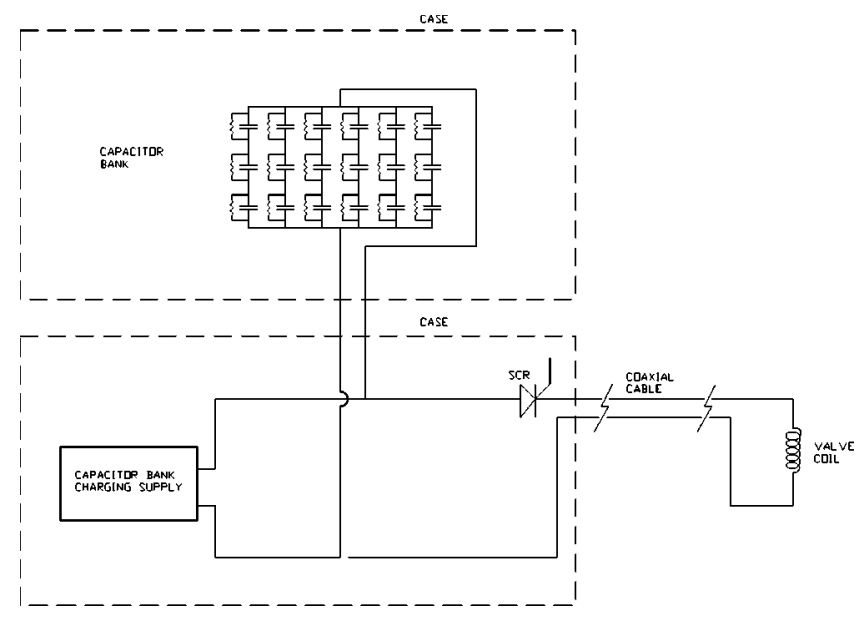

FIG. 4. Electrolytic capacitors in separate case from charging supply, meter, and SCR: this arrangement eliminates external heating of the capacitors and, by switching capacitors, allows for a comparison of electrolytic with metallized polypropylene film capacitors (the latter not shown here).

\section{COMPARISON BETWEEN ELECTROLYTIC AND METALLIZED POLYPROPYLENE FILM CAPACITOR BANKS}

In order to confirm that the problem was indeed due to the temperature dependence of the electrolytic pulse capacitors a special test configuration was constructed. The electrolytic capacitors were placed in a separate case so that any change in capacitor temperature would be entirely due to self-heating or dissipation by the bleeder resistors; this arrangement is sketched in Fig. 4. An identical case was constructed with metallized polypropylene film energy storage capacitors having the same capacitance (two $200 \mu \mathrm{F}$ capacitors in parallel, CSI Technologies Model 01CT0007). Both cases had a type $\mathrm{N}$ coaxial panel jack which was used to connect to the charging supply via a type $\mathrm{N}$ male-male adapter. Measurements were made with either the electrolytic or the metallized polypropylene film capacitors connected.

Figure 5 shows the behavior of the photoflash electrolytic capacitors for this test configuration. It is seen that the temperature rise when the photoflash electrolytic capacitors are in a separate case is about $3{ }^{\circ} \mathrm{C}$ compared to the $\sim 10^{\circ} \mathrm{C}$ temperature rise when they are in the same case as the charging supply. In Fig. 5, the charging supply was turned off during the $4 \mathrm{~h}$ wait times after shots 50, 100, and 150 whereas for the data in Fig. 3, the charging supply was left on.

The dependence of capacitor bank performance on prior temperature history as well as firing sequence is evident from comparing Fig. 3 to Fig. 5. In Fig. 3 (capacitor bank left charged during $4 \mathrm{~h}$ wait times), there is an upward jump in temperature, current, and pressure on going from shot 50 to shot 51; this can be attributed to the slow heating of the case and capacitors while the capacitors are in standby at $400 \mathrm{~V}$ and the power supply is heating the case. However, in going from shot 100 to shot 101 in Fig. 3, the temperature of $32^{\circ} \mathrm{C}$ is above the standby equilibrium temperature so the system cools during the $4 \mathrm{~h}$ wait and the current and pressure decrease. In Fig. 5, the capacitors cool in the $4 \mathrm{~h}$ intervals between shots 50 and 51, 100 and 101, and 150 and 151

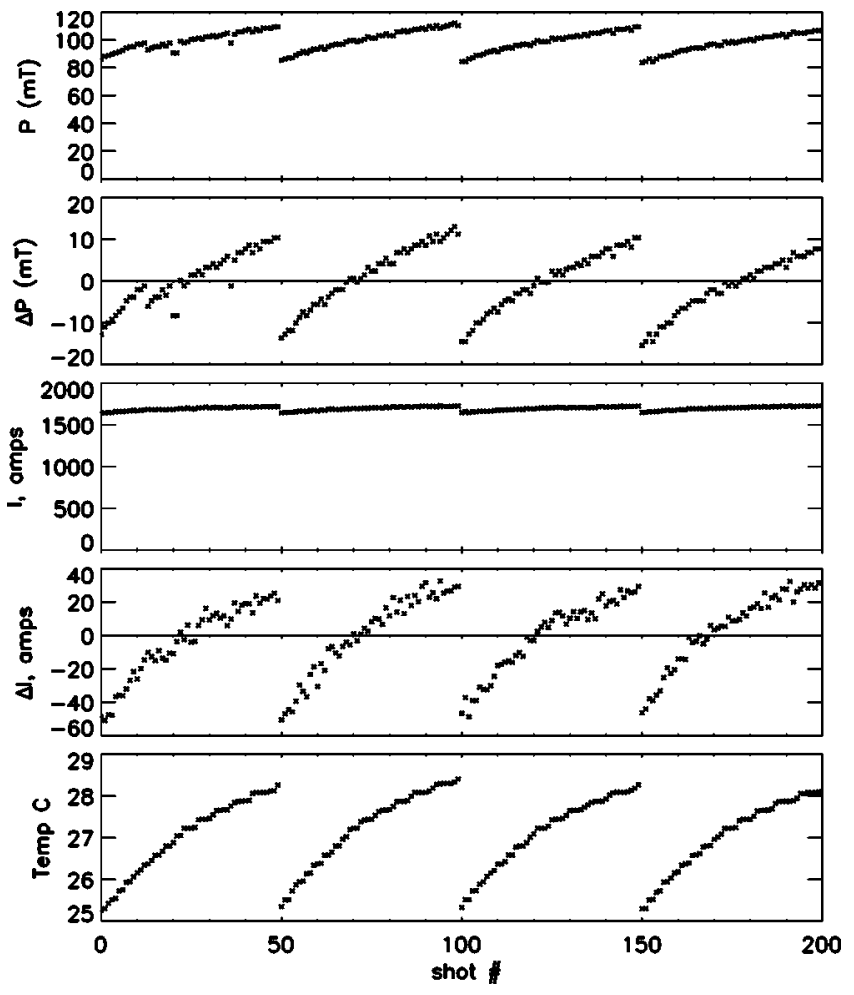

FIG. 5. Electrolytic capacitors in separate case from charging supply, meter, and SCR: 200 shot sequence with same cadence and charge voltage as in Fig. 3 except that capacitors are not charged during four hour wait periods.

because in this sequence the capacitors are held at $0 \mathrm{~V}$ during the $4 \mathrm{~h}$ wait. Figures 3 and 5 show that there is a strong dependence of capacitor bank peak current on the temperature of the electrolytic capacitor bank and that the temperature is a function of both the cadence at which the bank is pulsed and the heat input during standby.

Figure 6 shows data taken using the $400 \mu \mathrm{F}$ metallized polypropylene film capacitors. A thermocouple attached to the side of one of the metallized polypropylene film capacitors monitors capacitor temperature. The pressure pulse with the metallized polypropylene film capacitor bank was found to be much higher than that of the electrolytic bank when charged to $400 \mathrm{~V}$ (750 $\mathrm{mT}$ versus the range of $80-250 \mathrm{mT}$ ). This increased pulse was presumably due to the lower ESR of the metallized polypropylene film capacitors so that a larger fraction of the energy stored in the capacitor was coupled to the gas valve. The charging voltage was reduced to $220 \mathrm{~V}$ in order to obtain a pressure rise similar to that provided by the photoflash electrolytic capacitors. Figure 6 shows that there is a very small temperature rise and minimal shot-to-shot variation in output current or pressure for the metallized polypropylene film capacitors. The rms shot-toshot pressure variation is $1.5 \%$ and the rms shot-to-shot current variation is $0.3 \%$. The voltage waveform of the metallized polypropylene film capacitors showed that this configuration is underdamped and has a $25 \%$ voltage reversal (43 $\mu$ s half period); in contrast, the voltage waveform of the photoflash electrolytic capacitors is overdamped (14 $\mu \mathrm{s}$ full width at half maximum).

Because the discharge time for the polypropylene capacitors was so much longer and the required charge voltage 


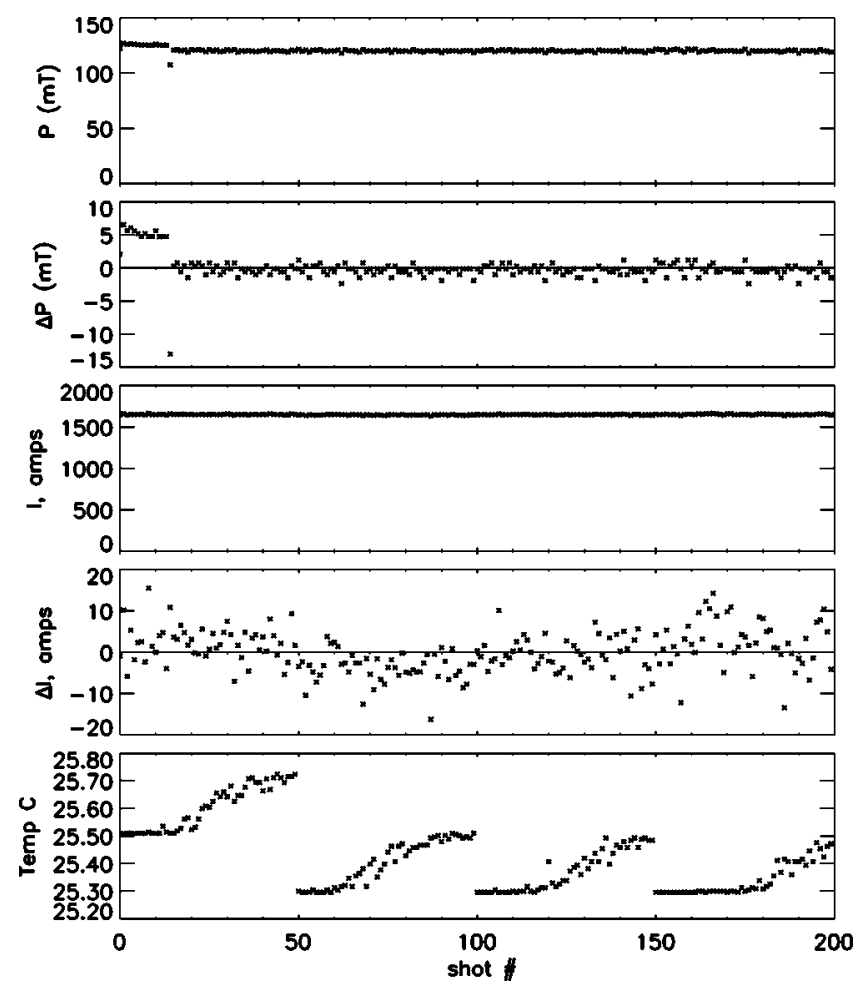

FIG. 6. Metallized polypropylene film capacitors, $400 \mu \mathrm{F}$, in separate case from charging supply, meter, and SCR: all settings are the same as for Figs. 3 and 5 except that charge voltage has been reduced to $220 \mathrm{~V}$ and capacitors are not charged during $4 \mathrm{~h}$ wait periods.

was so much smaller than the electrolytic capacitors, it was decided to use a polypropylene capacitor with smaller capacitance, but operating at higher voltage in order to shorten the waveform. A $50 \mu \mathrm{F}$ metallized polypropylene film capacitor (CSI Technologies Model 01CT0025) charged to 625 $\mathrm{V}$ provided an $\sim 130 \mathrm{mT}$ pressure rise and had a $50 \%$ voltage reversal. In order to avoid energy transfer back to the capacitor via ringing and so minimize capacitor heating, a crowbar diode was added consisting of four International Rectifier $1600 \mathrm{~V}$ diodes, Model $85 \mathrm{HF} 160$ in parallel. The rms variation in pressure rise over 200 shots is $0.8 \%$ for the noncrowbar case and $0.5 \%$ for the crowbar case. Figure 7 shows results obtained for the $50 \mu \mathrm{F}$ metallized polypropylene capacitor circuit (with crowbar diode) charged to $625 \mathrm{~V}$. This highly reproducible performance $(0.5 \% \mathrm{rms}$ variation in pressure rise over 200 shots) is an enormous improvement over the huge (i.e., 250\%) variation in pressure rise obtained using the photoflash electrolytic capacitors (compare Fig. 7 to Fig. 3).

\section{DISCUSSION}

This investigation shows that charging an electrolytic capacitor bank to a precise voltage does not guarantee that the energy output will be precise or reproducible. At least some of the irreproducibility in previous spheromak experiments is likely due to this subtle thermal sensitivity of the gas valve system (or of other systems involving pulsed electrolytic capacitor banks). Because heat is deposited inside a capacitor on each pulse and then slowly diffuses to the capacitor surface, active cooling of the capacitor surface is unlikely to

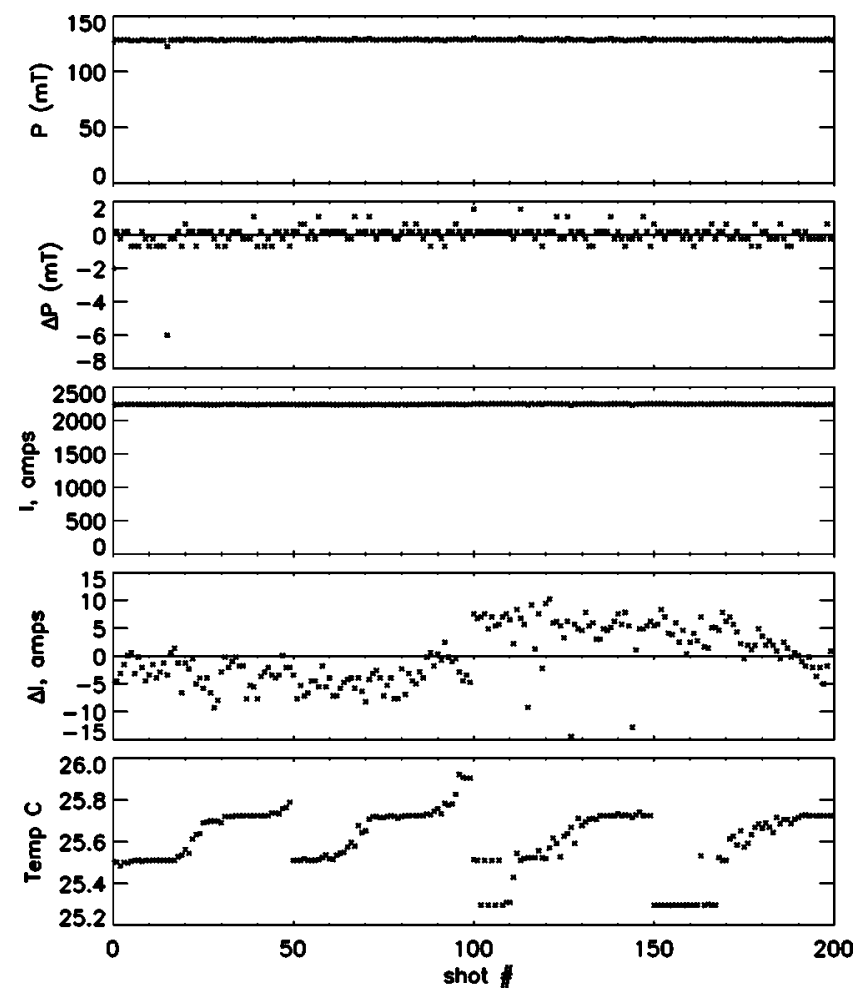

FIG. 7. Metallized polypropylene film capacitor, $50 \mu \mathrm{F}$, in separate case, all settings same as for Figs. 3, 5, and 6 except that charge voltage has been increased to $625 \mathrm{~V}$, capacitors are not charged during $4 \mathrm{~h}$ wait periods. Valve coil has a crowbar diode in parallel to eliminate power ringing back into the capacitor. Note large improvement in reproducibility of pressure rise compared to Figs. 3 and 5 (i.e., $0.5 \%$ rms variation in pressure rise here compared to $250 \%$ increase in Fig. 3, 30\% increase in Fig. 5).

cure this reproducibility problem except in the unlikely situation where the pulse repetition rate is kept absolutely constant so that the system can settle into a perfectly reproducible thermal cycle. We conclude from the thermal drift problem identified here that electrolytic capacitors should not be used in high current, pulsed power applications where high reproducibility is required unless means can be found to compensate for the extreme temperature sensitivity of the equivalent series resistance of these capacitors.

\section{ACKNOWLEDGMENTS}

The author thanks F. C. Cosso and D. D. Felt for their assistance in constructing the various versions of the capacitor bank. This work was supported by U.S. DOE Grant No. DE-FG03-98ER54461.

${ }^{1}$ J. C. Fernandez, C. W. Barnes, T. R. Jarboe, I. Henins, H. W. Hoida, P. L. Klingner, S. O. Knox, G. J. Marklin, and B. L. Wright, Nucl. Fusion 28, 1555 (1988).

${ }^{2}$ M. R. Brown and P. M. Bellan, Phys. Rev. Lett. 64, 2144 (1990).

${ }^{3}$ R. Raman, F. Martin, B. Quirion, M. Stonge, J. L. LaChamber, D. Michaud, B. Sawatzky, J. Thomas, A. Hirose, D. Hwang, N. Richard, and C. Cote, Phys. Rev. Lett. 73, 3101 (1994).

${ }^{4}$ H. S. McLean, D. Q. Hwang, R. D. Horton, R. W. Evans, and S. D. Terry, Fusion Technol. 33, 252 (1998).

${ }^{5}$ J. Yee and P. M. Bellan, Nucl. Fusion 38, 711 (1998).

${ }^{6}$ C. G. R. Geddes, T. W. Kornack, and M. R. Brown, Phys. Plasmas 5, 1027 (1998).

${ }^{7}$ J. F. Hansen and P. M. Bellan, Astrophys. J. 563, L183 (2001). 
${ }^{8}$ S. C. Hsu and P. M. Bellan, Monthly Notices R. Astron. Soc. (to be published).

${ }^{9}$ P. M. Bellan, Spheromaks (Imperial College Press, London, 2000).

${ }^{10}$ A. Von Engel, Ionized Gases (Oxford University Press, Oxford, 1965), p. 195.

${ }^{11}$ B. Gorowitz, K. Moses, and P. Gloersen, Rev. Sci. Instrum. 31, 146 (1960).

${ }^{12}$ G. Kuswa, C. Stallings, and A. Stamm, Rev. Sci. Instrum. 41, 1362 (1970).

${ }^{13}$ W. R. Gentry and C. F. Geise, Rev. Sci. Instrum. 49, 595 (1978).

${ }^{14}$ C. J. Keyser, M. Dembinski, and P. K. John, Rev. Sci. Instrum. 51, 425 (1980).

${ }^{15}$ S. Wong, P. Smiley, J. Levine, and V. Buck, Rev. Sci. Instrum. 57, 1684 (1986).

${ }^{16}$ J. H. Degnan, W. L. Baker, S. W. R. Warren, D. W. Price, M. P. Snell, R.
J. Richtersand, and P. J. Turchi, J. Appl. Phys. 61, 2763 (1987).

${ }^{17}$ O. F. Hagena, Rev. Sci. Instrum. 62, 2038 (1991).

${ }^{18}$ M. A. Sanner and J. Y. Park, Rev. Sci. Instrum. 67, 3675 (1996).

${ }^{19}$ T. Auguste, M. Bougeard, E. Caprin, P. D'Oliveira, and P. Monot, Rev. Sci. Instrum. 70, 2349 (1999).

${ }^{20}$ Y. Song, P. Coleman, B. H. Failor, A. Fisher, R. Ingermanson, J. S. Levine, H. Sze, E. Waisman, R. J. Commisso, T. Cochran, J. Davis, B. Moosman, A. L. Velikovich, B. V. Weber, D. Bell, and R. Schneider, Rev. Sci. Instrum. 71, 3080 (2000).

${ }^{21}$ I. Henins (private communication).

${ }^{22}$ J. C. Thomas, D. Q. Hwang, R. D. Horton, J. H. Rogers, and R. Raman, Rev. Sci. Instrum. 64, 1410 (1993).

${ }^{23}$ G. T. Smedley, D. J. Phares, and R. C. Flagan, Exp. Fluids 26, 116 (1999).

${ }^{24} \mathrm{R}$. Raman (private communication).

${ }^{25}$ J. Yee, Ph.D. thesis, Caltech, Pasadena, 2000, p. 92. 